\title{
The Method of \\ Archimedes: Propositions 13 and 14
}

\section{Shirley B. Gray, Daniel Ye Ding, Gustavo Gordillo, Samuel Landsberger, and Cye Waldman}

$\mathrm{N}$ $\mathrm{O}$ area of mathematics has attracted more international attention in the past decade than the Palimpsest of Archimedes. The 1998 auction at Christie's, followed by collaborative work centered at the Walters Art Museum led to traveling museum exhibits, newspaper articles, television specials, and dozens of presentations. Mathematicians and other scholars attracted a new and significant audience. The singed, battered, faded, mildewed, damaged 10th century manuscript-the world's oldest copy of The Method of Archimedes-sold for \$2 million "under the hammer." Mathematicians and classical scholars have long wondered just how close Archimedes (287-212 BC), a mechanical genius, had come to formulating modern calculus. The clues would surely lie in Propositions 13 and 14, if only they could be read. Though now transcribed, the content may contain copyists' errors. In the

Shirley B. Gray is professor of mathematics at California State University, Los Angeles. Her email address is sgray@exchange.calstatela.edu.

Daniel Ye Ding is an undergraduate student at California State University, Los Angeles. His email address is ddanie1497@gmai1. com.

Gustavo Gordillo is a graduate student at California State University, Los Angeles. His email address is miztag@hotmai 1 . com.

Samuel Landsberger is a professor at California State University, Los Angeles. His email address is slandsb@ calstatela.edu.

Cye Waldman is a consultant and National Curve Bank contributor. His email address is cye@att. net.

For permission to reprint this article, please contact: reprint-permission@ams.org.

DOI: http://dx.doi.org/10.1090/noti1279
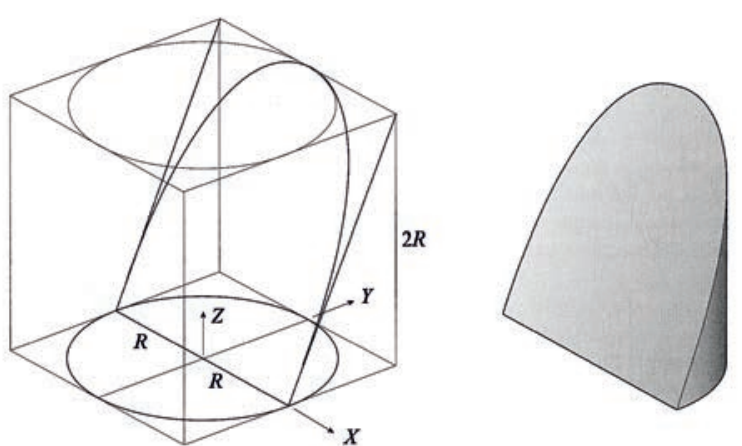

Figure 1. Development of the hoof.

true Archimedean experimental tradition, we decided to avail ourselves of the opportunity to look not retrospectively at the content of propositions in The Method but rather in terms of 21 st century mathematics and technology. Moreover, we believe we participated in every scholar's quest to have a Eureka moment-we found the Golden Ratio in our attempts to image the footprint of Archimedes.

\section{Volume of the Cylinder "Hoof"}

The cylinder hoof (a.k.a. "ungula", Latin for hoof) has been an object of fascination since Archimedes demonstrated, by geometric means alone, that the volume is one-sixth that of an enclosing cube. In the present effort, we show how to calculate the volume of the hoof by contemporary means, utilizing trigonometry and calculus, both of which were unknown to Archimedes in the third century BC. 


\section{Technical Discussion}

The hoof is created by "cuts" on a cube. The sequence as in Figure 1 (left) has been demonstrated by several scholars over the past century [1-11]. We begin with a cube; the first cut leaves behind a cylinder whose diameter is equal to the side of the cube; the hoof is created by cutting the cylinder with a plane that bisects its base and passes through its upper lip. The final hoof is shown in Figure 1 (above).

As we shall be using trigonometry and calculus, we need to clearly define the coordinate system and dimensions (as shown in Figure 1). A cube of side $2 R$ is centered on the $X-Y$ plane ( $X$ and $Y$ each vary from $-R$ to $R$ ) and has a height of $2 R$, i.e., $Z$ varies from 0 to $2 R$. Thus, the enclosed cylinder has a radius of $R$. For definiteness, we take the slant cut to intersect the $X$-axis. The height and base of the hoof both are $2 R$.

Clearly, the volume of the hoof is then

$$
V=\int_{-R}^{R} \int_{0}^{\sqrt{R^{2}-X^{2}}} \int_{0}^{2 Y} d Z d Y d X
$$

Now, the volume of an object is a physical property. So imagine that we needed to solve Eq. (1) numerically for many radii; we would have to solve each case separately. Rather, let's reduce it to a purely mathematical (and dimensionless) problem as follows: let $x=X / R$, and similarly for $Y$ and $Z$. Then Eq. (1) becomes

$$
V=R^{3} \int_{-1}^{1} \int_{0}^{\sqrt{1-x^{2}}} \int_{0}^{2 y} d z d y d x
$$

Now we can see that the volume scales exactly as $R^{3}$ and we can consider the purely numerical (i.e., dimensionless) problem that can be solved once, and for all $R$.

$$
V=\int_{-1}^{1} \int_{0}^{\sqrt{1-x^{2}}} \int_{0}^{2 y} d z d y d x
$$

This equation may easily be solved without Mathematica ${ }^{\circledR}$ or MATLAB. Let's break it down as follows:

(4)

$$
\begin{aligned}
& V=\int_{-1}^{1}\left[\int_{0}^{\sqrt{1-x^{2}}}\left[\int_{0}^{2 y} d z\right] d y\right] d x \\
& \int_{0}^{2 y} d z=2 y \\
& \int_{0}^{\sqrt{1-x^{2}}} 2 y d y=\left.y^{2}\right|_{0} ^{\sqrt{1-x^{2}}}=1-x^{2} \\
& V=\int_{-1}^{1}\left(1-x^{2}\right) d x=\left.x\left(x-\frac{x^{3}}{3}\right)\right|_{-1} ^{1}=\frac{4}{3}
\end{aligned}
$$

Finally, since $V_{\text {cube }}=8 R^{3}$, it is apparent that $V_{\text {hoof }} / V_{\text {cube }}=1 / 6$, as was first determined by Archimedes over 2,000 years ago.

Lynch [29] has shown how to calculate the volume of the hoof by calculation of single integrals. As seen in Figure 2, the calculation can be done

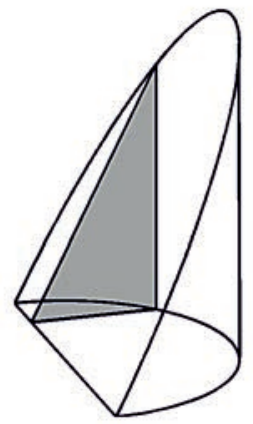

(A)

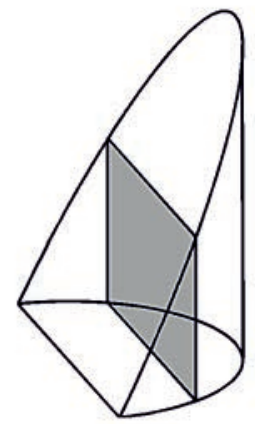

(B)

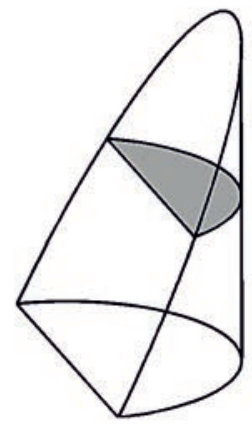

(C)
Figure 2. Cross-sections of a hoof in the $x, y, z$ directions [after Lynch (2012)].

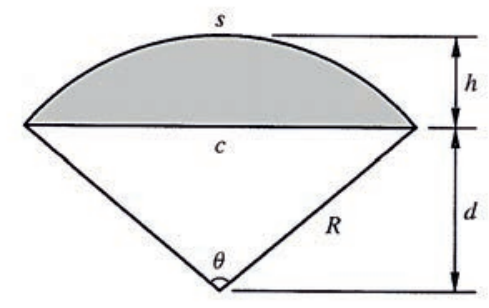

Figure 3. Segment of a circular section (shaded).

by integrating a triangle, rectangle, or segment in the $x, y$, and $z$-directions, respectively. All three integrations give the same result, of course: $V_{\text {hoof }}=4 R^{3} / 3$. We demonstrate these calculations below, in dimensionless form. The goal is to integrate each area over the appropriate coordinate; using the procedure for nondimensionalization of the coordinates, we have

$$
\begin{aligned}
v & =\int_{-1}^{1} A_{\text {triangle }}(x) d x \\
& =\int_{0}^{1} A_{\text {rect }}(y) d y=\int_{0}^{2} A_{\text {segment }}(z) d z .
\end{aligned}
$$

The first two areas are given by

$$
\begin{aligned}
& A_{\text {triangle }}(x)=\left(1-x^{2}\right) \\
& A_{\text {rect }}(y)=4 y \sqrt{1-y^{2}} .
\end{aligned}
$$

It can be shown that each of these integrals is given by $v=4 / 3$, in agreement with Eq. (4). The area of a circular segment, as shown in Figure 3, is given by

$$
A_{\text {segment }}(y)=\cos ^{-1} y-y \sqrt{\left(1-y^{2}\right)} \text {. }
$$

However, we wish to integrate this function over $z$; noting that $z=2 y$ and letting $\zeta=z / 2$, we can 

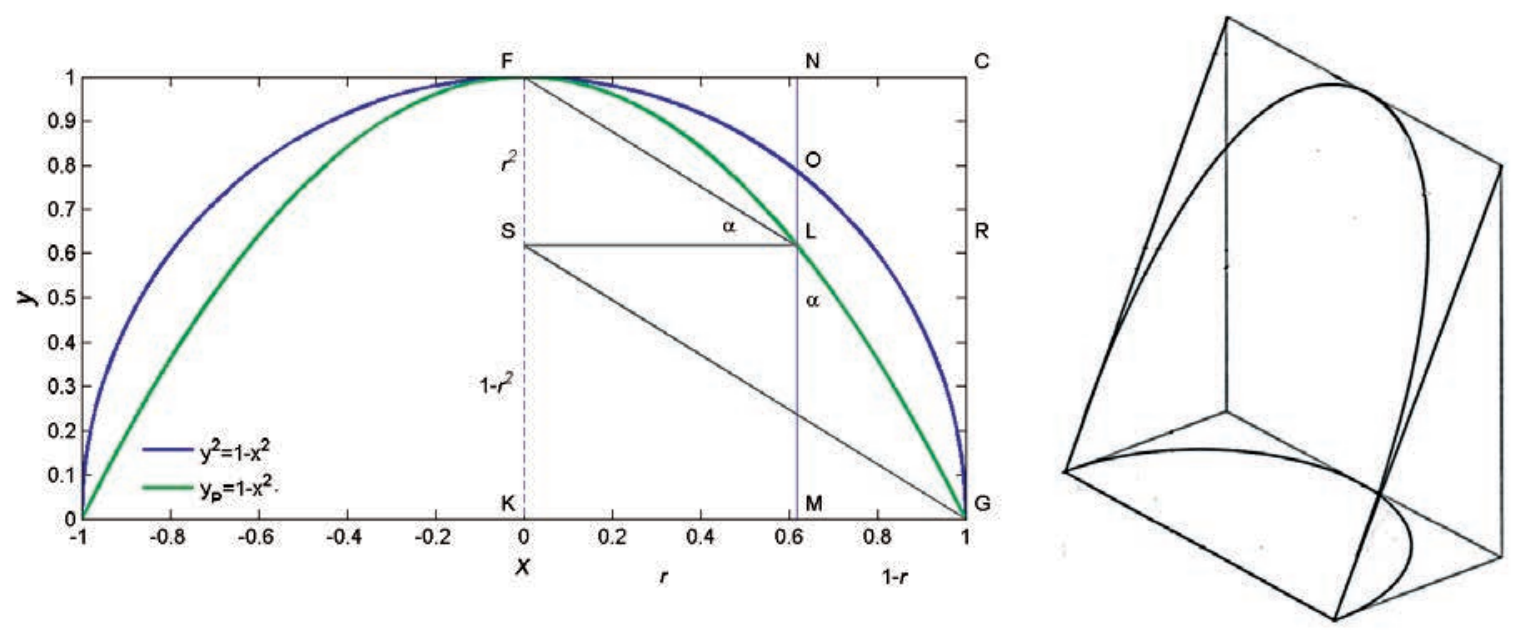

Figure 4: The footprint of the hoof and the parabola of Archimedes (left) and the hoof and prism

(right).

write

$$
\begin{aligned}
v & =2 \int_{0}^{1} A_{\text {segment }}(\zeta) d \zeta \\
& =2\left[\zeta \cos ^{-1} \zeta-\sqrt{1-\zeta^{2}}+\frac{1}{3} \sqrt{\left(1-\zeta^{2}\right)^{3}}\right]_{0}^{1} \\
& =\frac{4}{3}
\end{aligned}
$$

In closing, let us reflect for a moment on how Archimedes solved this problem. He was essentially using the triangular method described above, but lacked the tools that are at our disposal. Archimedes considered the problem of summing the triangles in the $x$-direction to obtain the volume, i.e., that which we have expressed as

$$
v=\int_{-1}^{1} A_{\text {triangle }}(x) d x=\int_{-1}^{1}\left(1-x^{2}\right) d x .
$$

Archimedes demonstrated in his Proposition 13 that the integrand in this equation, which derives from the circle, $y^{2}=1-x^{2}$, is also the equation of a parabola in the $x-y$ plane, $y_{p}=1-x^{2}$, as seen in the green line in Figure 4 (above). In the notational form of ratio and proportion used by Archimedes, $\mathrm{MN}^{2}: \mathrm{MO}^{2}:: \mathrm{MN}: \mathrm{ML}$. Thus, the (dimensionless) volume of the hoof is equal to the (dimensionless) area of the parabolic segment! Now, this might seem confusing because we appear to have lost a dimension. That is an artifact of the nondimensionalization. In the dimensional world, this would be expressed in terms of ratios. Figure 4 (right) shows the volumes of the hoof and prism. The volume of the prism is to that of the hoof as the area of the base rectangle is to that of the parabolic segment, i.e.,

$$
V_{\text {prism }} / V_{\text {hoof }}=A_{\text {rect }} / A_{\text {par }} \text {. }
$$

Archimedes had previously shown that the area of the rectangle to that of the parabolic segment is $A_{\text {rect }} / A_{\text {par }}=3 / 2$. And noting that $V_{\text {prism }} / V_{\text {cube }}=$ $1 / 4$, we again have $V_{\text {hoof }} / V_{\text {cube }}=1 / 6$.

Notably, we believe we participated in every scholar's quest to have a Eureka moment-we found the Golden Ratio in our attempts to image the footprint of Archimedes, but only when $r=\frac{R}{\varphi}$. In Figure 4 (above), note that $r$ varies from 0 to $R$. None of the other scholars cited in this manuscript has ever pointed out that if using the actual magnitude of NF or NL, you can readily show from the similar (in fact, congruent) triangles $\triangle \mathrm{GML} \sim \triangle \mathrm{LSF}$, that $r=\frac{1}{\varphi}$, the inverse of the Golden Ratio: ME:ED::MN:MK::NF:NL $=\varphi$, and incidentally, $\mathrm{MO}=1 / \sqrt{\varphi}$.

\section{The Animation and Computer Program} Animations shown at curvebank.calstatela. edu/method/method.htm demonstrate the transition from cube to cylinder to hoof. Each is composed of vertical laminae of thickness $\Delta x$ that make up the volume. In the final sequence of the animation, the triangles are stripped away from the hoof to show its internals; a dis-integration, if you will. The animation was created with a variant of the MATLAB program. Each of the laminae is built up of bands of width $\Delta x$; these are appropriately modified to avoid a staircase effect when they are stacked together. 
The code reports the ratio $V_{\text {cube }} / V_{\text {hoof }}$ and you can verify that this approaches 6 as the parameter npts increases (this essentially reduces $\Delta x$, the laminae thickness). For example, for npts $=51$ we obtain $V_{\text {cube }} / V_{\text {hoof }}=6.0024$ whereas for npts $=501$ we get $V_{\text {cube }} / V_{\text {hoof }}=6.000024$. Close enough for government work, as we say.

We also posit that a "hoof" similarly constructed from an arbitrary parallelepiped, thus composed of a slanted cylinder and elliptical cross-section, will similarly exhibit the property that the volume of the hoof is equal to one-sixth that of the parallelepiped. It all begins with a $4 \times 4$ matrix to map the points in the parallelepiped into a cube. We happily leave the details to the reader.

\section{3-D Printer Models}

Our team created two sets of models-an initial hoof on a smaller scale, and a medium-sized hoof, as well as its complement, on a larger machine. The smaller hoof, with a layer thickness of 0.01 inch, was made on a Deezmaker Bukobot printer. Its interior is practically hollow, and its surface walls have a thickness of 0.05 inch all around. The mediumsized hoof and its complement were printed using the Stratasys uPrint SE Plus on a thickness setting of 0.013 inch per layer. The dividing face common to both the hoof and its complement was omitted from their designs so that, like scoops, the final physical models can be filled with liquid. The printing processes were similar and began with computer representations of the geometric objects. In this case, a popular CAD (computeraided design) program called SolidWorks was used to create computer representations of the medium-sized hoof and its complement, where the reference cube used was 10-inches long on each side (see Figures 5 and 6). The software was able to calculate properties such as the volume, surface area, and center of mass of each model, and by doing so, gave us an independent verification that the hoof is $1 / 6$ the volume of the cube.

\section{References}

[1] J. L. HEIBERG, Eine Neue Archimedeshandschrift, Hermes, Volume 42, 1907, pp. 235-303.

[2] J. L. HeIberg and H. G. Zeuthen, Eine Neuve Schrift des Archimedes, Verlag von B. G. Teubner, Leipzig, 1907, pp. 321-363.

[3] Thomas L. HeAtH, The Method of Archimedes: Recently Discovered by Heiberg, Cambridge, 1912.

[4] _ , The Thirteen Books of Euclid's Elements, Volumes I, II and III, Dover, 1956.

[5] Great Books of The Western World, Robert Maynard Hutchins, ed., Mortimer J. Adler, Ass. ed., II: Euclid, Archimedes, Apollonius of Perga, Nicomachus, Encyclopedia Britannica, 1952.

[6] E. J. DijKsterhuis, Archimedes with a new bibliographic essay by Wilbur R. Knorr, Princeton University Press, 1987.

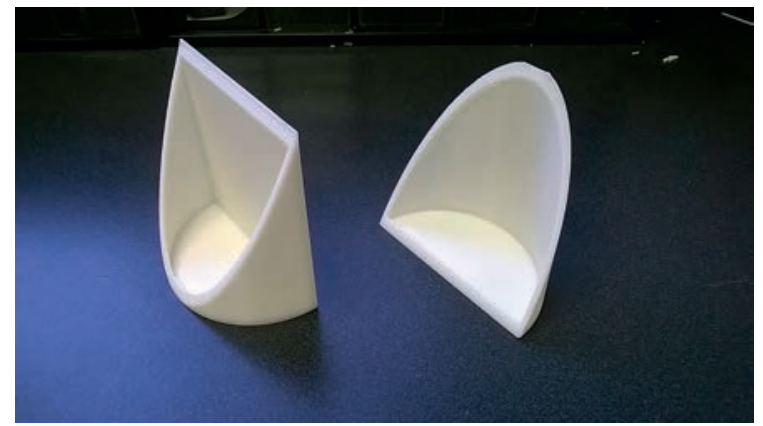

Figure 5.

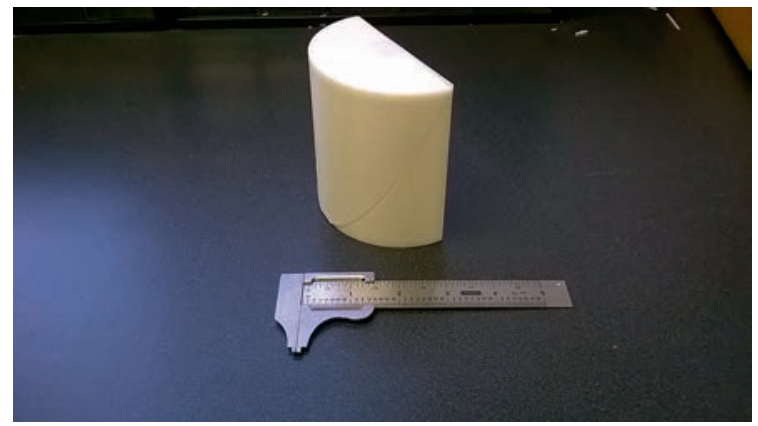

Figure 6.

[7] Reviel Netz, Ken Saito and Natalie Tchernetska, A New Reading of Method Proposition 14: Preliminary Evidence from the Archimedes Palimpsest (Part I), SCIAMSVS, Volume 2, April, 2001, pp. 9-29.

[8] _ , A New Reading of Method Proposition 14: Preliminary Evidence from the Archimedes Palimpsest (Part 2), SCIAMSVS, Volume 3, April, 2002, pp. 109-125.

[9] ReVIEL NeTZ, The Works of Archimedes, Volume 1, Cambridge, 2004.

[10] ReViel Netz and William NOEL, The ARCHIMEDES CODEX, Da Capo Press, 2007.

[11] ReViel NeTZ and William Noel, Natalie Tchernetska and Nigel Wilson, The ARCHIMEDES Palimpsest, Walters Art Museum and Cambridge, Volumes I and II, 2011.

[12] Sherman Stein, Archimedes: What Did He Do Besides Cry Eureka, Mathematical Association of America, 1999.

[13] The Archimedes Palimpsest, Christie's (Auction catalog, October 29, 1998), 502 Park Avenue, New York, 10022.

[14] S. H. Gould, The Method of Archimedes, American Mathematical Monthly, Volume 62, 1955, pp. 473-476.

[15] J. L. HeiberG, translated by D. C. Macgregor, Mathematics and Physical Science in Classical Antiquity, Chapters in the History of Science II, Charles Singer, ed., Oxford, 1922.

[16] Archimedes Text Sold for \$2 Million, The New York Times, October 30, 1998, p. A22.

[17] CARol Vogel, Eureka, The New York Times, February 19, 1999. 


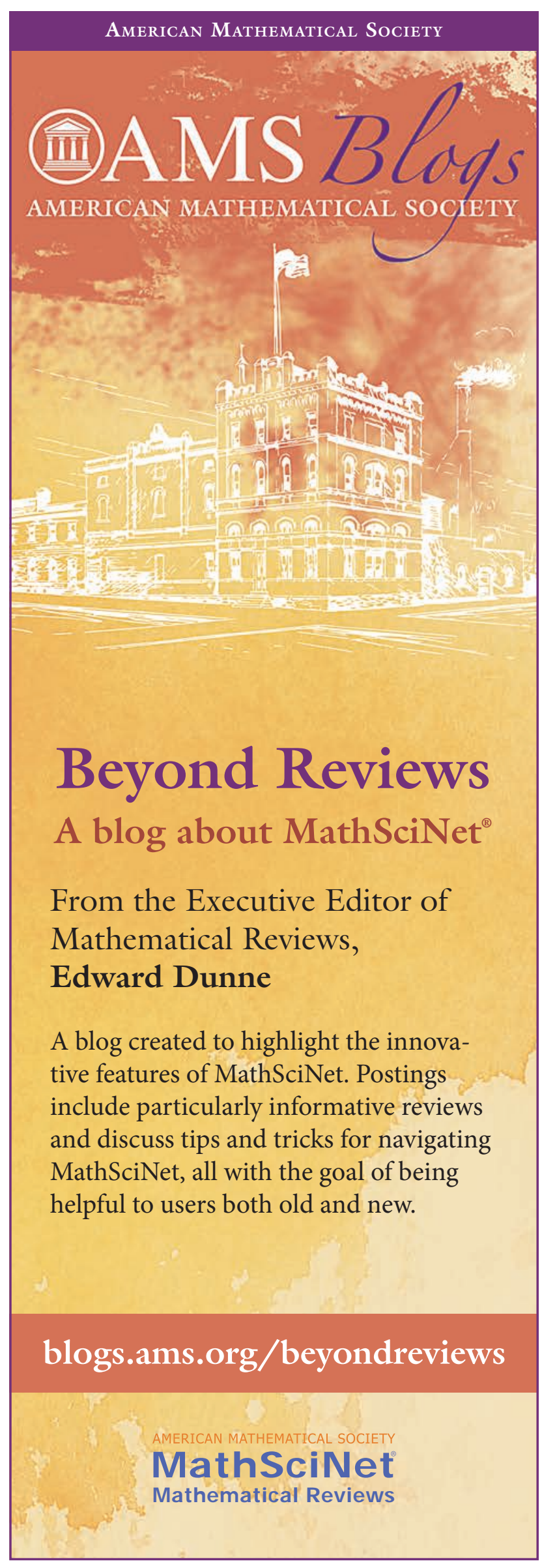

[18] Big Literary Find in Constantinople, The New York Times, July 16, 1907, p. 1.

[19] Archimedes text goes to unnamed buyer for $\$ 2.2 \mathrm{~m}$, Kathimerini, Athens, October 30, 1998, p. 1.

[20] Half-leaf of a 10th century codex, translated by Nigel Wilson, Cambridge University Library, Add.1879.23.

[21] W. R. KNORR, The Method of Indivisibles in Ancient Geometry, Vita Mathematica (R. Calinger, ed.,) Mathematical Association of American, 1996, pp. 71-74.

[22] C. H. EDWARDS JR., The Historical Development of the Calculus, Springer-Verlag, 1979, pp. 68-76.

[23] Archimedes Comes to America, Science, Volume 284, June 25, 1999, p. 2083.

[24] IVAN BoSERUP (ed.), Københavns Universitet 14791979: Klassisk filologi efter 1899, Volume 8, 1992, pp. 367-374.

[25] Shirley B. Gray, A Centennial Celebration of Two Great Scholars, Notices of the American Mathematical Association, Volume 55 (7), August, 2008, pp. 776-783.

[26] J. L. Berggren, The Archimedes Codex, Book Review, Notices of the American Mathematical Association, Volume 55 (8), September, 2008, pp. 943-947.

[27] James Stewart, Calculus, 5ed, Thomson, Brooks/Cole, 2003, p. 888.

[28] AMIr AleXANDER, Infinitesimal, Scientific American / Farrar, Straus and Giroux, 2014, pp. 107-111.

[29] P. LYNCH, "Sharing a Pint" ThatsMaths thatsmaths .com/2012/12/13/sharing-a-pint/.

[30] PBS (2003) Infinite Secrets, NOVA Science Programming, Original PBS Date: September 30, 2003.

\section{Websites}

curvebank. calstatela.edu/method/method. htm

www.cs.drexe1.edu/ crorres/Archimedes/ contents.html

www youtube . com/watch? $v=u w i d w 12 k c t I$

www. archimedespalimpsest.org/

web.calstatela.edu/faculty/hmendel/

Ancient\%20Mathematics/Archimedes/

Archimedes\%20Method/Prop14/Arch.Method.

Prop.14.htm1 